\title{
Intelligence and Intelligent Simulation
}

\author{
V.Yu. MEYTUS \\ International Research and Training Center for Information Technologies and Systems \\ of the National Academy of Sciences of Ukraine
}

\begin{abstract}
The work presents a new approach to the study of problems associated with the initial definition of the concept of "intelligence". In work, intelligence is a property that ensures, at some level, the successful interaction of a subject or system with their environment, which is specified in the form of a subject area. For this, the subject area is modeled (smart modeling). Modeling is the process of representing a domain in which the subject must solve the corresponding problems.

The domain model is presented as a body of knowledge covering the constituent elements of this domain. Knowledge is a means of describing this model and is determined using a language that includes the logic of describing the corresponding entities of the subject area. Language properties are used to model some abstract formal structure in the form of a mathematical representation. The modeling process consists in a sequential transition from the description of the subject area to an interconnected structure of knowledge, associated with the constituent elements of this area, which are highlighted in the modeling process.
\end{abstract}

Keywords. Intelligence, intelligent system, subject area, mathematical structure, logic, modeling, intelligent modeling, knowledge, knowledge representation, ontology.

\section{Introduction}

There are many science fiction novels and films, the heroes of which are machines created by man, but possessing reason and intellect. And therefore, they are capable of solving the most difficult problems on which the existence of mankind in the future depends, which way will its development go, how it will be able to protect itself from internal and external threats.

The real path to the creation of such machines began when computer systems emerged as the basis for collecting, accumulating, storing and processing data about the world around a person. With the help of these systems, first of all, they made an attempt to solve problems that a person successfully solves, relying on his knowledge and his intelligence, on his feelings and emotions. These were the tasks of recognizing signs, texts, images, competing with a person in various games - checkers, chess, poker, go - understanding what a person is talking about, translating from one language into another, writing poetry and creating musical works. In other words, we are talking about solving problems that are considered creative and have always been the prerogative of a person.

These tasks have historically been called artificial intelligence (AI) [1 - 3]. But, over time, this concept has expanded. The tools that simulated creative functions expanded their capabilities to new areas of application. Now AI is widely used in developments related to entire areas of human activity: industry, robotics, transport, 
medicine, defense, and education. AI has found applications in finance, trade, security systems, and data mining. Algorithms and methods related to AI are used wherever it is necessary to process large amounts of various data, identify existing dependencies and use them to optimize decisions.

At the same time, a new approach has been outlined for constructing systems that are supposed to have intelligence, and in their direction of research differ from classical AI. The basis of this direction is the definition of intelligence as a certain property, the presence of which in a subject or system means that such a subject or system can be called intellectual. The definition of intelligence simultaneously shows that there are different representations for intelligence, although they all have some common properties.

This work is devoted to the study of the main properties and possibilities associated with this new approach. Its purpose is to highlight and detail the basic elements that collectively define intelligence. In particular, this element is smart modeling, discussed below.

\section{Basic concepts}

\subsection{Intelligence.}

Consider the basic concepts related to intelligence. Firstly, any intelligence is related to and manifests itself in a certain subject area. The subject area is a part of the real, imaginary or in some other way a given world, environment, within a certain context..

It is assumed that any subject area can be described by a certain set of concepts, rules and restrictions that are specific to this area. Usually a person works in a certain field and, if you need to characterize him, then say a financier, mathematician, physicist, composer, programmer or athlete, thereby in a sense defining what a person does and where he manifests himself. Although in some cases a person can express himself in different subject areas. For example, the physicist A. Einstein played the violin well, and the politician W. Churchill painted beautiful pictures.

Note that along with the natural material world, considered as a set of subject areas - place of residence, home, study, work, entertainment (theaters, clubs, concerts) - one can consider the worlds of ideas, representations, fantasies, beliefs, hopes, expectations, virtual structures. Subject areas include the fields of science, art, management, and production.

In order to carry out any actions in the subject area, you first need to set or describe these actions for yourself. For this, there is some representation of the subject area, called its model. In this model, actions are formally performed. And intelligence is used in the context of this model. Therefore, the second necessary property of intelligence is the ability and ability to adequately model the subject area, based on their perception of the individual components of this area. The process of modeling itself, since it involves a connection with intelligence, is called intelligent modeling.

Finally, the last third property of intelligence, in which it manifests itself most vividly, is the solution of problems in the subject area. First, the problem is solved in the constructed model, and then, for implementation in reality, it is transferred to the subject area, in which the elements of the model are interpreted. Success in solving problems depends on how adequate the model of the domain was built, and on whether 
the intellectual subject will be able to find a solution to problems using the constituent elements of the model.

Let's combine these properties in a single concept. Then the intellect of the subject is understood as a property that allows the subject to model the subject area, which he perceives and with which he interacts, so that at the level of the constructed model to solve the problems that can be posed to the subject in this area. [4]

A system with intelligence, that is, capable of simulating its environment and solving problems on the basis of this model, is called an intelligent system. Note that it is possible to consider systems for which a domain model is specified, and the system only solves problems based on this model. For example, this is how the teaching of mathematics at school is structured: textbooks contain information and rules, the use of which allows you to solve problems associated with these rules.

Subject area modeling. Let the subject area $\mathcal{A}$ be given, for which an intelligent system (IS) $\mathfrak{I}$ is being built. This system is created to organize interaction with $\mathcal{A}$. The interaction itself is determined by the tasks coming from $\mathcal{A}$ to system $\mathfrak{I}$, and the solutions of these problems by $\mathfrak{I}$. It is assumed that the system $\mathfrak{I}$ forms for itself some idea of the subject area - the model of area $\mathcal{A}$ - on the basis of information flows coming from $\mathcal{A}$ to $\mathfrak{I}$. These streams are formed using the means of perception that the IC has, or other means used by the IC to characterize the area $\mathcal{A}$.

Model $\mathcal{A}$ is a part of a certain space that includes a partially named set $\mathrm{U}$ of objects and structures of the area $\mathcal{A}$, together with the connections and relations existing in this area, perceived by the IS and reflected by it in the model.

The set $\mathrm{U}$ is called the universe $\mathcal{A}$. Partial naming implies that individual elements of $\mathcal{A}$ or their constructions from these elements can have their own name. Concepts are associated with them as a description of a set of properties characteristic of this part. Behind the concepts are the classes of objects that exist in $\mathcal{A}$.

Concepts $(\mathrm{N})$, individual representatives of the class of objects (Id) defined by concepts, attributes of concepts (M), relations $\mathrm{R}$ in the aggregate make up the universe $\mathrm{U}$, and hereinafter are called concepts of model $\mathcal{A}$. A variant of perception of the area $\mathcal{A}$ is possible not immediately, but gradually. For example, with the help of a training sequence, the elements of which form separate concepts that define the characteristics $\mathcal{A}$. In this case, the IS does not even directly perceive the subject area. All the necessary information is contained in the training sequence.

In addition to the formation of a set of concepts at the stage of modeling, the IS chooses the type of spaces (mathematical structures) in which the model will be built. These can be algebraic structures, topological spaces, manifolds, categories, functional spaces, and neural networks of various shapes, probabilistic models and networks, fuzzy spaces, automata. The features of the selected space are used in the construction of the model, being a constituent part of it. Let's call the selected structure the bearing mathematical structure of the domain model.

The second component of the understanding of intelligence is the logic $\mathcal{L}$, which underlies both the construction of a domain model and the solution of problems that are posed to the IS. In the general case, logic, as a means of formalizing reasoning, shows how, in the condition of certain assumptions, from some statements - the conditions of the problem - it is possible to obtain other statements, considered as a result of solving this problem. But classical logic is not well suited for this. Therefore, in theory of modelling, an attempt was made to expand the possibilities of logical inference, improving it and bringing it closer to the methods with which a person solves problems. 
To build IS, one can use, for example, predicate logic, modal and fuzzy logics, evolutionary variants of logic, default logic, probabilistic logic, and descriptive logic. In each case, the system receives its own version of intelligence with its own problemsolving capabilities.

\subsection{Knowledge.}

In order to build a model of a subject area, IS collects information about the structure, constituent elements, connections between the elements of this area, IS collects information about the structure, constituent elements, connections between the elements of this area, the possibility of transforming some elements into others.. Moreover, this information comes in separate portions and is stored in the system, forming in it an idea of the subject area in the form of a body of knowledge. [4, 5]

Each knowledge is generated by an objective reality in which an IP (subject) exists. But this reality is transformed through the perception of the IP (subject). Or it is set ready-made, as a result of the perception of other subjects. The process of knowledge formation can be empirical: the collected information is brought together and transformed into the form of knowledge. Or theoretical, passing first through the creation of a theory that is valid in a certain area, and only then including theoretical hypotheses and conclusions in the form of knowledge about the environment. Although between these forms of obtaining knowledge, in reality, transitions and interconnections are possible and exist.

Based on the collected knowledge, the IS performs modeling. The process of creating a model can be one-time: " $\{$ knowledge $\} \rightarrow$ model", or sequential and multiple, in which knowledge is gradually built up and a new amount of knowledge allows you to form a new approximation of the domain model.

Naturally, the collected knowledge should contain enough information to serve as the basis for a domain model, and be consistent with the capabilities of IP. It is proposed to consider the multicomponent task of knowledge, which from different sides define the object to which the knowledge is compared. In the future, this knowledge can be used to build a model.

Let's choose an arbitrary object $\mathrm{X}$ belonging to the area $\mathcal{A}$. Perceiving this object, the IS forms a certain set of features $\alpha^{X}=\left(\alpha_{1}^{X}, \ldots, \alpha_{n}^{X}\right)$, built on the basis of this perception. Signs can be as simple as estimating the size or color of an object. And they can be complex if the object is associated with some concepts of the subject area, for example, a tree, a garden, a stream, and a ravine. Suppose that the set $\alpha^{X}$ is the value of some operator $\Lambda$ on the object $\mathrm{X}$. And this value $\Lambda(\mathrm{X})$ is one of the components of knowledge $\varsigma_{X}$ about the object $\mathrm{X} . \Lambda(\mathrm{X})$ is called the natural interpretation of the object X.

The second component of knowledge is built on the basis of logic $\mathcal{L}$. This component consists of a logical description of the object $\mathrm{X}$ in the form of a formula in the language of logic $\mathcal{L}$. This formula will be denoted as $\mathcal{L}(\mathrm{X})$. In area $\mathcal{A}$, object $\mathrm{X}$ is linked to other objects from $\mathcal{A}$ by different relationships and dependencies. It is assumed that the set of such objects associated with $X$ is finite. Such a set of pairs (object $\mathrm{Y}$, relation $\mathrm{R}$ ), for which object $\mathrm{Y}$ is connected with object $\mathrm{X}$ by relation $\mathrm{R}$ in $\mathcal{A}$, constitutes the third component of knowledge about object $\mathrm{X}$. Finally, the last fourth component of knowledge is the ontological description of object $\mathrm{X}$. The use of 
ontology is a prerequisite for human understanding of knowledge representation. What cannot be described at the level of ontology is not knowledge for a person either.

Note that, in the general case, ontology can include various means of description for different subject areas: the language of mathematical theory and chemical formulas, mythological representations, metaphors, fantastic images and descriptions. Interpretation of an object from $\mathcal{A}$ in the form of an ontological description is the basis on which the interaction of the IS with a person is further built.

\subsection{Intelligent Modeling Algorithm.}

The concepts discussed above are combined in a basic algorithm for constructing a domain model. A variant of such an algorithm was proposed in [6].

The intelligent modeling process is structured as follows.

1. The supporting mathematical structure [6], considered as the skeleton of the model, is selected as the basic basis for the created model. The choice of the supporting structure largely determines the methods that can be used in the model to find solutions to problems. The components of this structure in the subject area are compared to entities that the IS (subject) perceives, separates from the area and finds for them a possible set of properties (features, attributes).

2. Entities in the subject area include:

- objects perceived at this level as some kind of integrity;

- instances of objects;

- classes of objects;

- structures built from various objects, classes;

- connections and relationships between objects, classes, structures;

- a set of objects considered as a single entity;

- processes considered as a sequential change in a set of objects.

It is assumed that each of these entities can be perceived and separated from the subject area for modeling. Moreover, some entities that have the same or similar properties can be combined into a common class. Finally, an entity can be formed during the modeling process when other entities are selected, or it can arise based on the choice of an ontology associated with the subject area.

Note that other approaches to considering entities in the subject area are also possible. For example, when considering different variants of neural networks as a carrier structure, sets of weights of individual neuron configurations can be considered as entities.

To accumulate experience of its work, the IS contains a knowledge base in which various variants of subject areas and their components are presented and accumulated in the course of work. The intelligent modeling algorithm assumes the possibility of accessing this database. The request is based on the information that the subject endows with the entities selected from the subject area. At the same time, the possible environment of the entity in question can be selected from the base.

3. After building a domain model, intelligence appears as the basis for solving problems in this model. The supporting mathematical structure associated with the subject area sets the core of a formal approach to solving future problems, to the choice of the necessary methods and an abstract approach to the presentation of information that needs to be used in the solution. In turn, the logic associated with intelligence sets the rules on the basis of which the IS will seek a solution to the tasks assigned to it. 
4. Logic is the formal basis for solving future problems. It coincides with the logic that is chosen to build the domain model. The logical description of knowledge is one of the components of its presentation. And logical view processing is an integral part of the problem-solving path. Above, we have considered possible options for specifying logic that can be used to describe knowledge and the subsequent conclusion of consequences when solving a problem. In fact, there are much more possible options for specifying logic. And research in this direction is constantly ongoing. In many respects, the efficiency of the intellect in solving the problem, as well as the complexity of the problems being solved, depends on the task of logic.

5. The intelligent modeling process usually includes several stages. In other words, the domain model is not created immediately as a one-time sequential execution of the first four steps of the overall building process. As practice shows, first, based on the selected set of entities, the first approximation of the model is built. It is then analyzed and viewed as a representation of the domain for comparison and validation of the model and the domain. It is possible that the constructed model reflects only some characteristics of the region and requires clarification of the elements presented in the model. Then the modeling process is reapplied to the pair (subject area, model). The constructed model is expanded and refined; new elements and entities are introduced into it so that it more adequately reflects the subject area.

6. The finished model is immersed in the IP knowledge base. In the future, many of these models determine the experience of an intelligent subject or system, making it possible to simplify the modeling process. Therefore, the process of sequential building of the model is transformed into the process of its training.

7. Along with the process of intelligent modeling and consideration of its result the model of the subject area - the knowledge base includes information related to the choice of the supporting mathematical structure and the choice of the logic of description and construction of the model. Such a choice can be determined by analogy with ready-made solutions or randomly. But determining the nature of the supporting structure and the logic of solving problems in a specific subject area is one of the complex unsolved problems associated with the development of the theory of intelligent systems.

\section{Conclusion.}

The work gives a definition of intelligence as a property that provides, at some level, the successful interaction of a subject or system with their environment in the form of a subject area. The system (subject) possessing this property is then called intellectual. The property of intelligence is expressed in the fact that such a system models a subject area, and then uses this model in order to solve problems in the model that determine the interaction "system $\leftrightarrow$ area".

The intelligent modeling algorithm selects for building a model some supporting mathematical structure, which is associated by the system with the perceived structure of the subject area. And to solve problems in the model, it uses one of the possible variants of logic. Naturally, for the same subject area, different versions of intelligences can be built, which have different capabilities, determined by the classes of problems to be solved. 
How for a specific area to choose the most suitable type of intelligence corresponding to it remains an open problem, which can be solved only after building different intelligences and comparing them in solving problems.

\section{References}

[1] Russell, S.J. Norvig, P. Artificial Intelligence: A Modern Approach (4nd ed.), Prentice Hall, 2020.$1115 \mathrm{p}$.

[2] Luger, G., Stubblefield, W. Artificial Intelligence: Structures and Strategies for Complex Problem Solving (5th ed.), Benjamin/Cummings Publishing Company, Inc., 2004.- 720 p.

[3] Hlybovets MM, Oletsky OV Artificial intelligence: a textbook. - K .: "KM Academy", 2002. 366 p.

[4] Meytus V. Problems of building intelligent systems. Knowledge representation. Cybernetics and Systems Analysis. 2019, №4. p. 3-14.

[5] Meytus V. Introduction to the theory of intelligent systems. Basic views. Saarbrücken: Palmarium academic publishing, 2015. $189 \mathrm{p}$.

[6] Meytus V. Problems of building intelligent systems. Intellectual simulation. Cybernetics and Systems Analysis. 2021, №4. p.3-19. 